\title{
1097 Automated frame-by-frame endocardial border detection from cardiac magnetic resonance images for quantitative assessment of left ventricular function: validation and clinical feasibility
}

\author{
Cristiana Corsi*1, Federico Veronesi ${ }^{1}$, Claudio Lamberti ${ }^{1}$, Dianna ME Bardo ${ }^{2}$, \\ Ernest B Jamison ${ }^{2}$, Roberto M Lang ${ }^{2}$ and Victor Mor-Avi ${ }^{2}$
}

Address: ${ }^{1}$ University of Bologna, Bologna, Italy and ${ }^{2}$ University of Chicago, Chicago, IL, USA

* Corresponding author

from I th Annual SCMR Scientific Sessions

Los Angeles, CA, USA. I-3 February 2008

Published: 22 October 2008

Journal of Cardiovascular Magnetic Resonance 2008, I0(Suppl I):A222 doi:10.I I86/I532-429X-I0-SI-A222

This abstract is available from: http://jcmr-online.com/content/I0/SI/A222

(c) 2008 Corsi et al; licensee BioMed Central Ltd.

\begin{abstract}
Introduction
Cardiac magnetic resonance (CMR) quantification of left ventricular (LV) size and function requires detection of endocardial boundaries. Most current techniques use image intensity gradients that require manual corrections and are thus subjective and time-consuming. As a result, clinically, CMR has been mostly used to measure only end-systolic and end-diastolic volumes (ESV, EDV) and calculate ejection fraction (EF).
\end{abstract}

\section{Purpose}

Our aims were: (1) to determine to what extent the lack of dynamic information affect LV volume and EF measurements, (2) to develop and test a technique for dynamic endocardial border detection independent of intensity gradients, as a basis for automated volume measurements throughout the cardiac cycle; (3) to validate this approach against manually traced endocardial boundaries, and test its clinical feasibility in patients with abnormal systolic and diastolic function.

\section{Methods}

35 patients (age $53 \pm 16$ yrs; 19 males) with a wide range of $\mathrm{LV}$ volumes $(39 \div 420 \mathrm{ml})$ and $\mathrm{EF}(20 \div 79 \%)$ were studied in two separate protocols. LV short-axis images (Philips $1.5 \mathrm{~T}$ ) were obtained from base to apex (30 frames/cardiac cycle) and analyzed using custom soft- ware. For each frame, 2D slices were stacked and endocardial boundaries automatically detected throughout the cardiac cycle in all slices simultaneously to preserve spatial continuity by using a modified 3D region-based model based on the probability of density distribution of gray levels. In protocol 1, we studied 19 patients. In addition to the automated analysis, endocardial contours were semi-automatically traced using commercial software (Philips ViewForum) as a reference for comparison. For both techniques, cross-sectional LV cavity area was calculated for each slice, and LV volume calculated throughout the cardiac cycle. Validation included inter-technique comparisons of LV volumes and EF. Border positions were compared by calculating mean radial distance between the automatically detected and the traced contours, normalized by mean contour radius. Also, slice areas were compared by computing the percentage of LV cavity area that did not overlap with the reference cavity area. Finally, several indices of systolic and diastolic function were calculated from each volume curve and compared between the two techniques. In protocol 2, we tested the clinical feasibility of the dynamic automated analysis of LV function in three groups: 14 patients with normal LV function, 12 patients with systolic dysfunction, and 10 patients with diastolic dysfunction secondary to LV hypertrophy. 

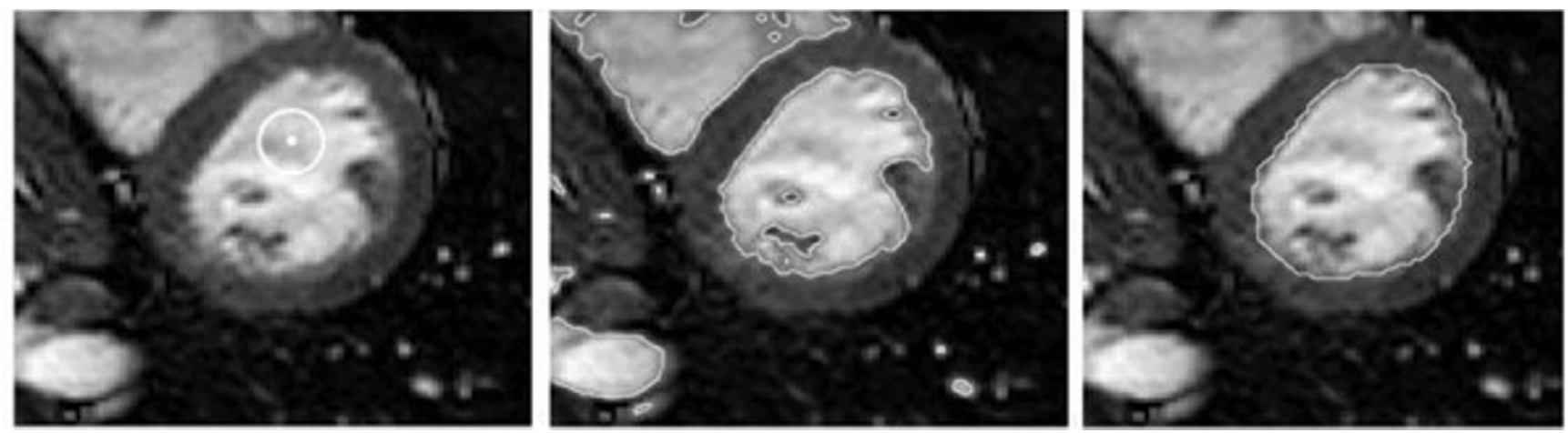

Figure I

We developed and tested a technique for endocardial border detection as a basis for automated frame-by-frame volume measurements. We validated this approach against manually traced endocardial boundaries, and tested its clinical feasibility in patients with abnormal systolic and diastolic function.

\section{Results}

Time required for automated analysis of one cardiac cycle was $<15$ minutes on a personal computer and resulted in endocardial boundaries that were judged accurate in all 35 patients (figure 1). In protocol 1, in 14/19 patients, dynamic analysis resulted in differences of at least one frame in the timing of end-systole and/or end-diastole compared to the conventional visual assessment, causing small but significant differences in $\operatorname{EDV}(+1.1 \pm 1.4 \%)$, ESV $(+2.3 \pm 1.9 \%)$ and EF $(-2.1 \pm 1.7 \%)$. Inter-technique comparisons showed high correlation coefficients and small biases (EDV and ESV: $\mathrm{r}=0.99$, bias $=-5.9 \pm 9.0 \mathrm{ml}$; EF: $r=0.96$, bias $=2.9 \pm 4.6 \%$ ). The differences in border position were: $9.6 \%$ and in slice cavity area $9.2 \%$, both differences being greater near the apex where endocardial boundaries are less defined. All calculated indices of LV systolic and diastolic function showed high inter-technique correlation (r: $65 \div 98$ ) and no significantly biases. In protocol 2, we found significant inter-group differences in multiple quantitative indices of systolic and diastolic function calculated from the LV volume time curves, indicating that our technique is sensitive enough to differentiate between these groups.

\section{Conclusion}

Fast, automated, dynamic detection of LV endocardial boundaries based on the probability of density distribution of gray levels is feasible and accurate. This approach allows accurate quantification of LV size and function, which is potentially clinically useful for objective assessment of systolic and diastolic dysfunction.

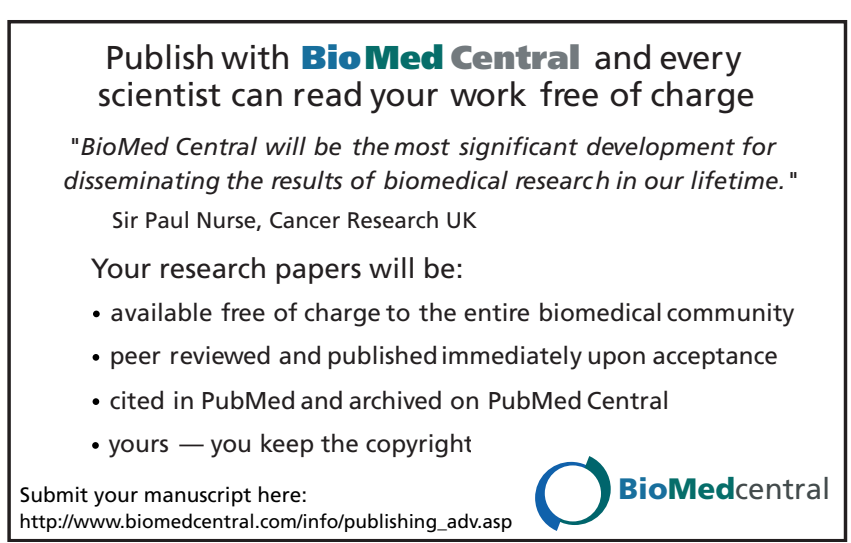

